\title{
Cardiac involvement in idiopathic haemochromatosis and the effect of venesection
}

\author{
S. J. JACHUCK \\ B.Sc., M.B.B.S. \\ G. S. RAI \\ M.B.B.S. \\ C. Fossard \\ M.B.B.S., M.Sc., M.R.C.P. \\ Department of Medicine, Newcastle General Hospital, Newcastle upon Tyne
}

Summary

Eight patients with idiopathetic haemochromatosis have been followed up for 2-15 years to study their cardiac state and the effect of repeated venesection in treating their disability. Five of these patients had no clinical cardiac disability. Serial electrocardiographs were done in five patients and significant abnormality was seen in three of them at the time of admission. After removal of excess iron the cardiac disability progressed in two patients and regressed in one other. One of these presented with palpitations and was found to have Wolff-Parkinson-White syndrome. Not one of our patients developed cardiac failure and the two deaths which occurred were the result of hepatic failure. At autopsy one of them was found to have metastases in the myocardium from a hepatoma, a complication we have not encountered in previous reports.

Correspondence: Dr S. J. Jachuck, Cruddas Park Medical Centre, Park Road, Newcastle upon Tyne NE4 7RZ
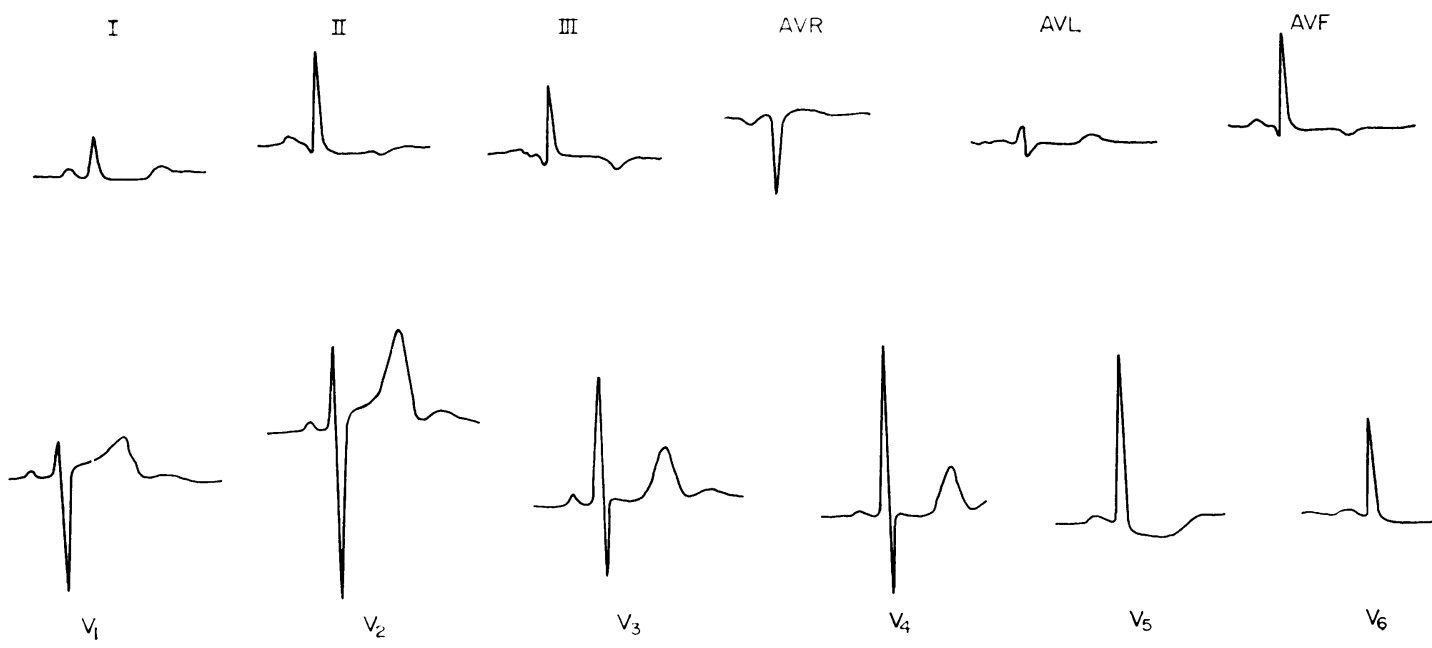

Cardiac failure, occasionally associated with cardiac dysrhythmias, is considered to be the commonest single cause of death in haemochromatosis (Truelove and Reynell, 1972) and accounts for about one-third of deaths (Thompson, 1969; Skinner and Kenmure, 1973). Reactionary fibrosis due to increased iron deposit in the parenchyma is a sequele of haemochromatosis and the aim of treatment is to deplete the body of excess iron. Removal of exces 8 iron from the body results in clinical, biochemica and histological improvement (Williams et al., 1969등. Although haemochromatosis usually manifests ilater life and is associated with diabetes mellitus, the cardiographic changes described so far are not related to coronary artery disease (Swan and Dewar, 1952).

Previous reports in the literature have often included patients with secondary haemosiderosis in addition to those with haemochromatosis.

This paper describes the cardiac involvement in

FIG. 1. ECG of patient W.W. showing ischaemic changes. 
idiopathic haemochromatosis and the effect of repeated venesection on the cardiac state. We use this data to support the view that cardiac failure is no longer the commonest cause of death in haemochromatosis (Williams et al., 1969).

\section{Methods and material}

The diagnosis of idiopathic haemochromatosis was made in eight patients, noting clinical manifestations of pigmentation and hepatic dysfunction associated with grade IV iron deposits in the liver parenchyma (Scheuer, Williams and Muir, 1962). In all cases the serum iron levels were greater than $300 \mu \mathrm{g} / \%$ with more than $80 \%$ saturation of transferin. The possibility of secondary haemosiderosis was excluded in each patient.

We describe the presenting complaint, the clinical manifestations of cardiac disability and electrocardiographic observation in five of them. Three other patients had no clinical evidence of cardiac disability and unfortunately electrocardiographic recordings were not carried out.

Each patient was treated by repeated venesection and to date two deaths have occurred. In both cases a hepatoma has been found at autopsy.

\section{Patient H.E.}

A 59-year-old man who was known to have had epilepsy for 39 years and diabetes for 4 years was admitted to this hospital in July 1966 when a diagnosis of haemochromatosis was made. At that time he denied any cardiac disability. An electrocardiogram was normal and the chest X-ray showed no cardiopulmonary abnormality. Venesection removing 500 $\mathrm{ml}$ blood on each occasion was commenced. After removing $30 \mathrm{~g}$ of iron the laboratory findings were as follows: haemoglobin, $14.9 \mathrm{~g} / \%$; serum iron, $214 \mu \mathrm{g}$; and iron binding capacity, $252 \mu \mathrm{g}$ with $85 \%$ saturation. At this time he denied any cardiac disability and his electrocardiogram showed sinus rhythm at a rate of $70 / \mathrm{min}$. Occasional supraventricular extra-systoles were seen. The $P$ wave was bifid and the duration was 0.08 . No other abnormality was seen.

\section{Patient $W . W$.}

A 53-year-old man presented with several years history of increasing pigmentation and listlessness. He was found to have haemochromatosis associated with a pre-diabetic glucose tolerance curve. He denied any cardiac disability and the electrocardiograph was normal. After removing $17 \mathrm{~g}$ of iron by repeated venesection his haemoglobin fell to $\mathbf{9 . 9}$ $\mathrm{g} / \%$ and the serum iron to $16 \mu \mathrm{g} / \%$ (iron binding capacity $399 \mu \mathrm{g} / \%, 4 \%$ saturation). His hepatomegaly noted on admission subsided and his symptoms
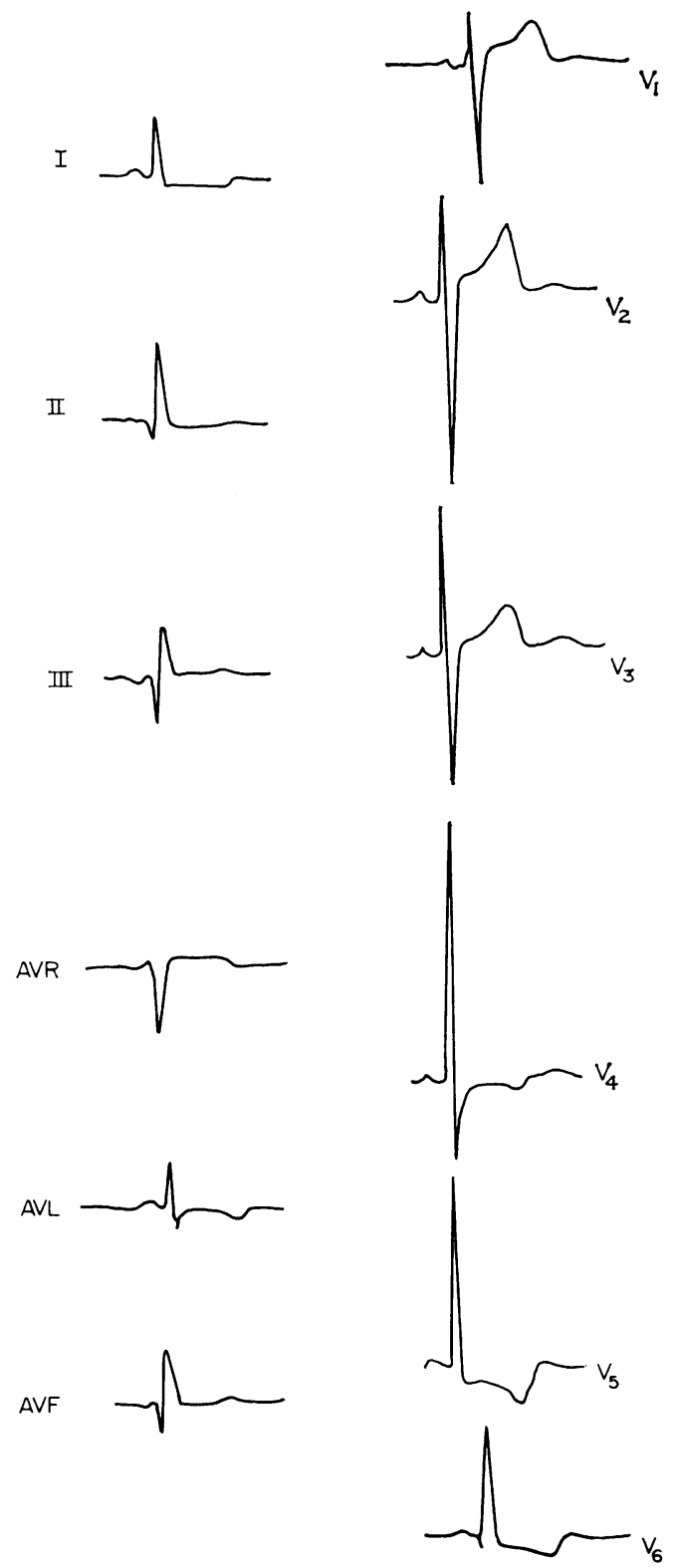

FIG. 2. ECG of patient W.W. 3 years later showing further ischaemic changes.

improved. Thirteen years later he developed angina of effort and exertional dyspnoea.

The electrocardiogram (Fig. 1) showed sinus rhythm at a rate of $55 / \mathrm{min}$. There were small $Q$ waves in leads II, III, AVF and the ST segment was depressed in lead I and V V-VII. No conduction defect was seen. Since then his cardiac disability has gradually deteriorated and the electrocardiogram shows progressive changes (Fig. 2). 


\section{Patient R.M.}

In October 1969, a 66-year-old man presented to this hospital with a 3 years' history of paroxysmal palpitations, exertional dyspnoea and one episode of paroxysmal nocturnal dyspnoea. He was found to have a diabetic glucose tolerance curve. The electrocardiogram at the time of diagnosis (Fig. 3) showed sinus rhythm at a rate of $72 / \mathrm{min}$. The $P$ wave was bifid and its duration was $0 \cdot 12 \mathrm{sec}$. The QRS configuration was compatible with Wolff-ParkinsonWhite syndrome. The ST segment was depressed in lead III and AVF. Inversion of T waves was seen in leads III, AVF and V I-III. No dysrhythmia was observed during his stay in the hospital. Despite repeated venesection, his cardiac disability increased. Nine months later he developed a junctional tachycardia with marked left axis deviation and ST depression (Fig. 4) suggesting further myocardial damage. His heart rate came down to $100 / \mathrm{min}$ with practolol but the junctional dysrhythmia persisted.

\section{Patient M.T.}

A 76-year-old man with diabetes treated with chlorpropamide was admitted for management of ascites when the diagnosis was made. His electrocardiogram (Fig. 5a, b) showed sinus rhythm with
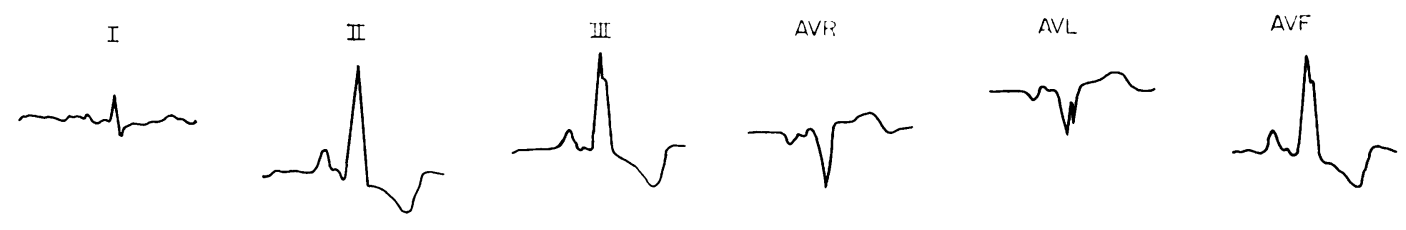
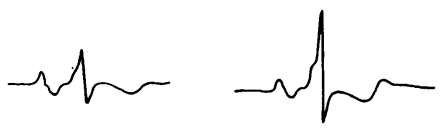

$V_{2}$

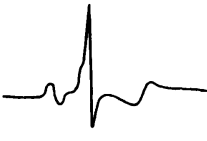

$V_{3}$

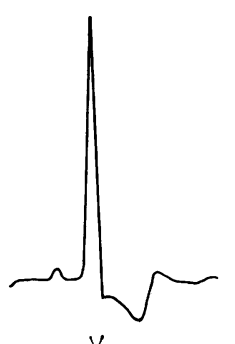

$v_{4}$

FIG. 3. ECG of patient R.M. showing Wolff-Parkinson-White syndrome.
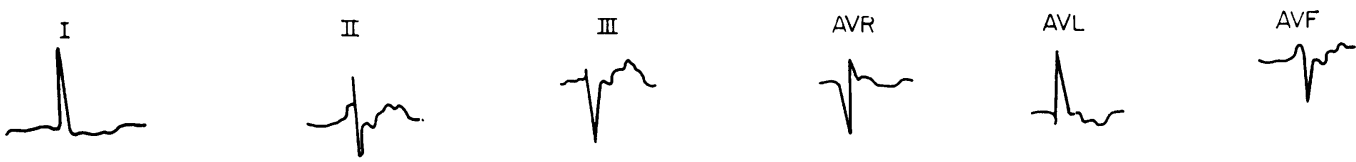

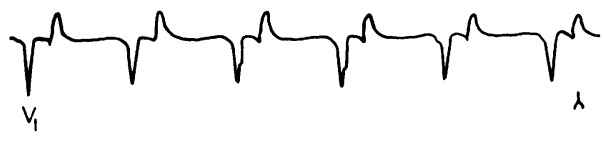
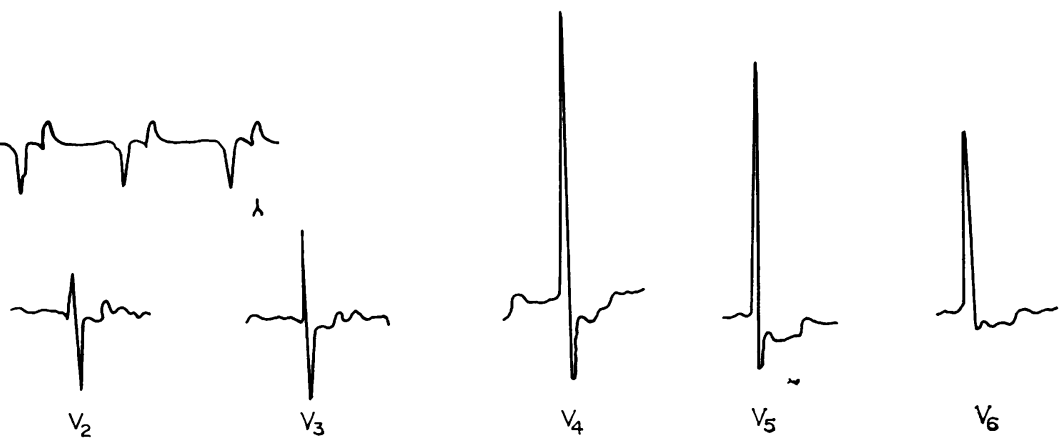

FIG. 4. ECG of patient R.M. 9 months later showing junctional tachycardia, left axis deviation and ST depression. 
(a)

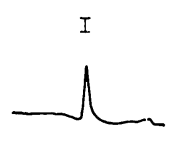<smiles>CCCCCC</smiles>

III

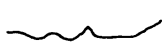<smiles>C=C=CCCC</smiles><smiles>CCCCCCC</smiles>
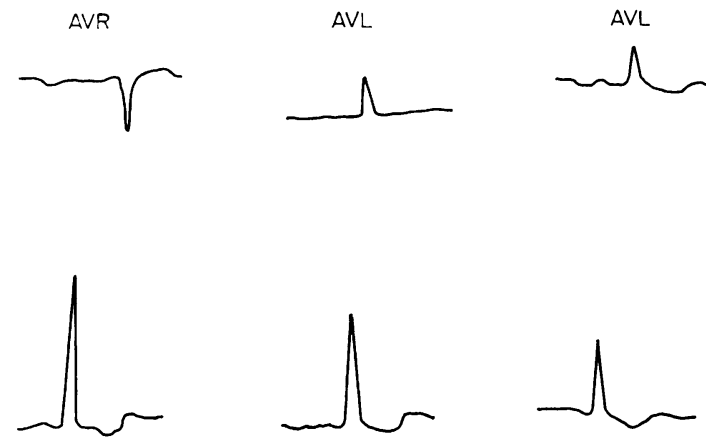

$V_{3}$

$V_{4}$

$v_{5}$
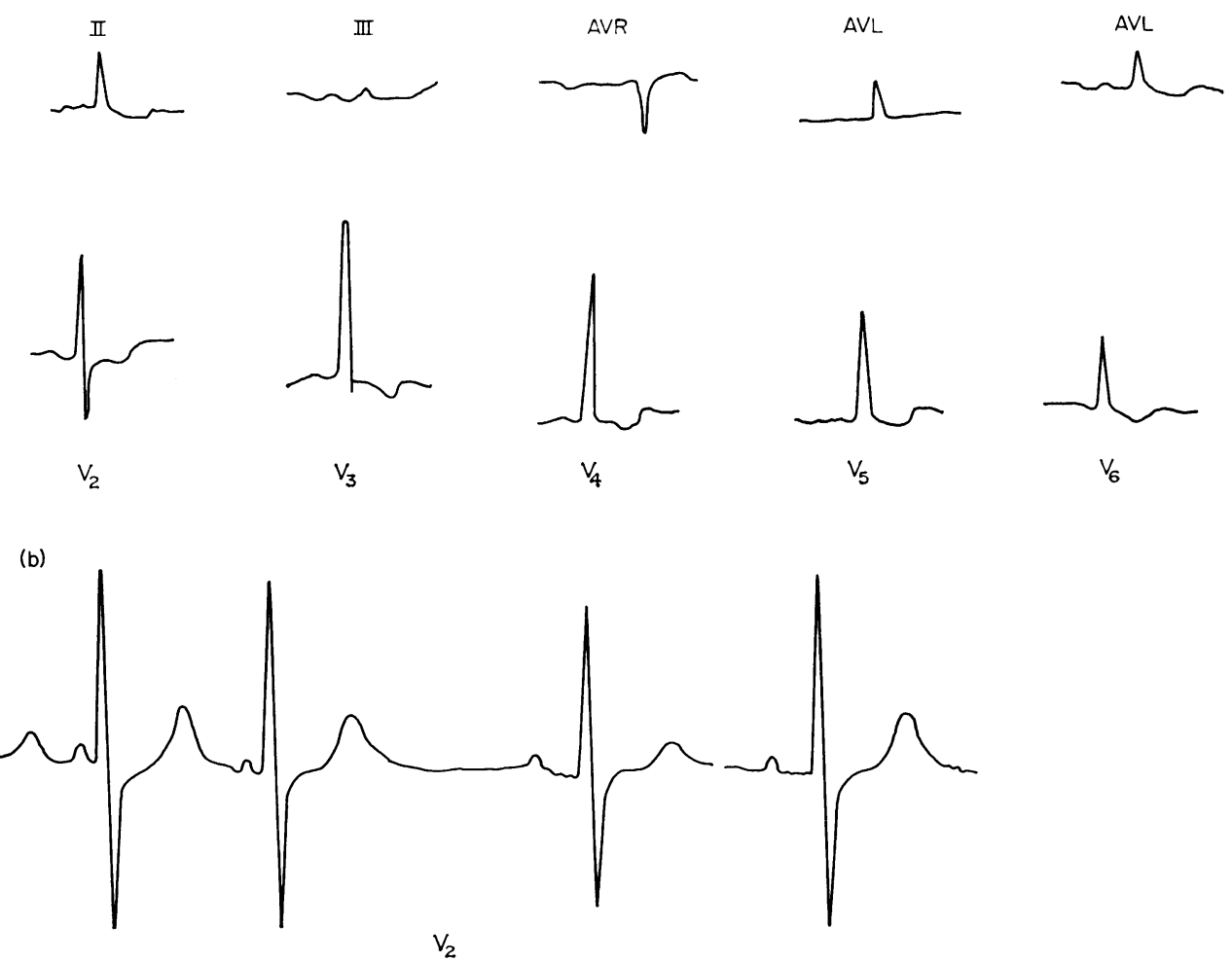

FIG. 5. Ischaemic changes (a) and rhythm strip (b) showing supraventricular extrasystole seen in M.T.
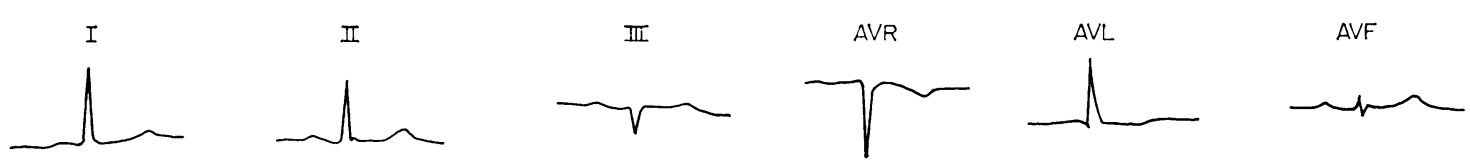

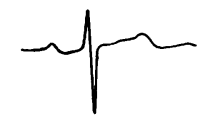

$V_{1}$
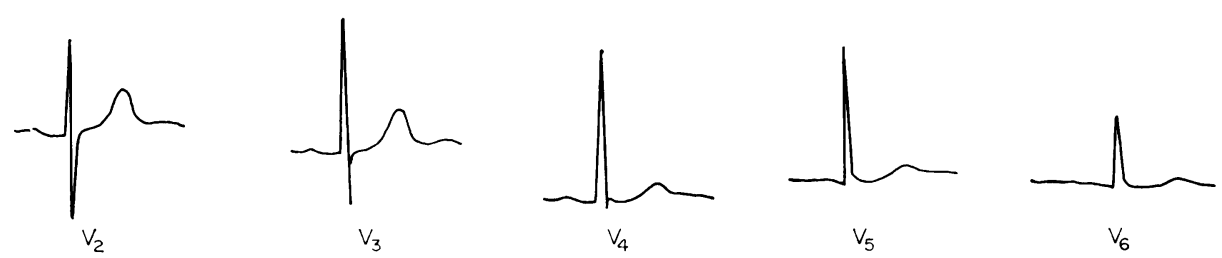

FIG. 6. ECG of patient M.T. after 16 months showing electrocardiographic improvement compared to Fig. 5.

supraventricular extra-systoles. $\mathrm{T}$ waves were inverted and there was no conduction defect. His general condition has improved considerably with venesection and diuretic therapy and his electrocardiogram (Fig. 6) shows improvement.

\section{Patient C.J.}

In 1964 a 57-year-old man with Parkinson's disease was admitted to one of the neurosurgical wards for stereotaxic surgery. He was found to have haemochromatosis with diabetes mellitus, and denied any cardiac disability. The electrocardiograph was normal apart from occasional ventricular extrasystoles. He was treated with carbohydrate restriction, insulin and repeated venesection. Two years later he was readmitted following the sudden onset 


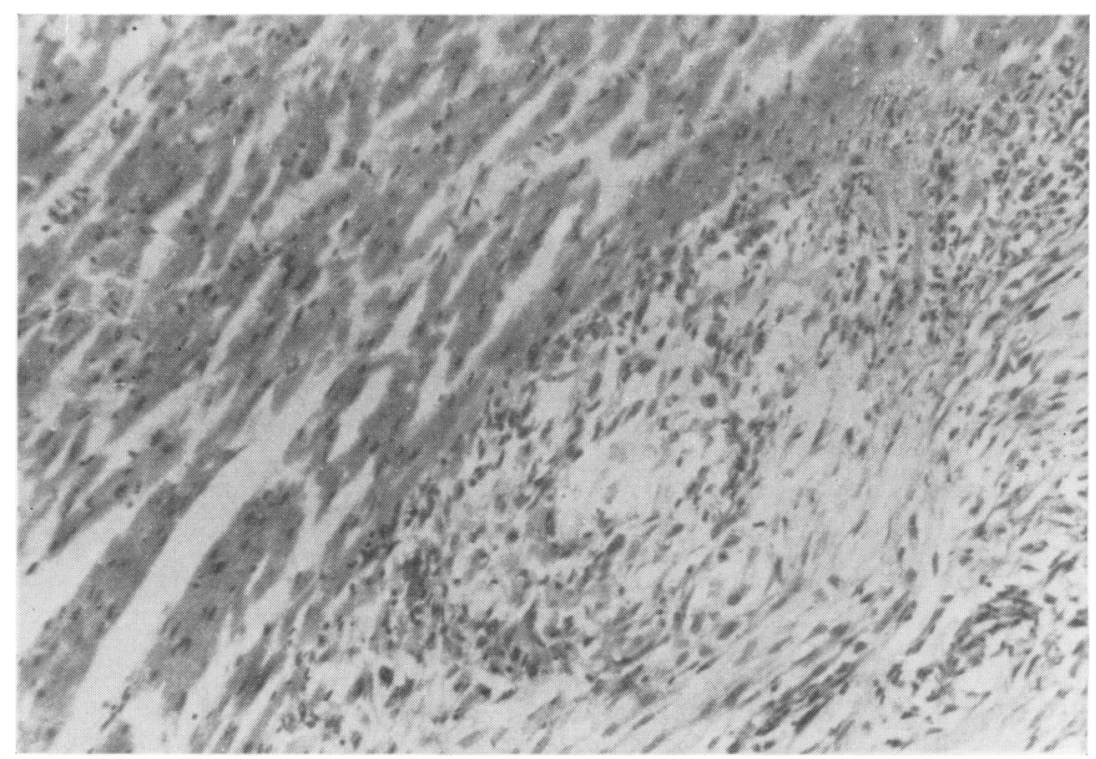

FIG. 7. Metastatic deposit in the wall of the left ventricle from a hepatoma.

of a right hemiplegia, and died 4 days later. His electrocardiograph showed no abnormality. At autopsy, haemochromatosis was confirmed and there were extensive metastases from a hepatoma. Three intramural metastatic deposits were found in the wall of the left ventricle (Fig. 7) and each measuring about $5 \mathrm{~mm}$ in diameter.

\section{Discussion}

The cardiac involvement in haemochromatosis and haemosiderosis is said to be the result of myocardial fibrosis. The electrocardiographic changes described include supraventricular as well as ventricular dysrhythmias, conduction defects, low voltage complexes and $\mathrm{T}$ wave changes (Swan and Dewar, 1952; James, 1964; Schellhammer, Engle and Hangstorm, 1966). One of the cases described by Swan and Dewar would appear to have suffered acute myocardial ischaemia or a sub-endocardial infarction. Although initially some patients have no cardiac disability, many of them eventually develop histological changes in the myocardium due to deposition of iron (Thompson, 1969), Schellhammer and his colleagues could find no correlation between the iron deposits found at autopsy and the antemortem conduction or rhythm disturbances; nor between the degree of scarring and the density of the iron deposits.

Two patients showed minor changes in the electrocardiographs. Patient C.J. showed occasional extrasystoles, not a useful indication of heart disease (British Medical Journal, 1973) and patient H.E. showed bifid $P$ waves. In patient C.J. autop revealed metastatic deposition of tumour cells in the left ventricle, but the coronary arteries were pateris Patients H.E. and M.R. showed bifid $P$ waves, sug $\vec{g}$ gesting left atrial hypertrophy, possibly the result of myocardial damage leading to left ventricular failure. No conduction defect was seen in any of these $\varrho$ patients, except for patient M.R. showing Wolff- $\overrightarrow{\vec{O}}$ Parkinson-White syndrome. This could be a coincidental association or may have developed secondary to damage of the atrioventicular node. Friedberg (1966) describes heart block as a common mani- $\bar{T}$ festation of cardiac involvement in this disorder. Absence of a conduction defect in these five cases may be due to arrest of the pathological process as a result of successful depletion of the excess iron. The low voltage electrocardiogram, as described by Swan and Dewar in two cases, was not seen in any of the patients described here. Dysrhythmias re- $\rightarrow$ corded in these five patients were supraventricular and ventricular extra-systoles and a junctional $N_{\sigma}$ tachycardia in a patient with Wolff-Parkinson-White syndrome.

None of these patients reported here suffered from $\mathrm{\omega}$ heart failure and the only two deaths in these eight patients were due to hepatic failure. Williams and $\stackrel{\circ}{工}$ associates have followed up fifty-eight patients with idiopathic haemochromatosis to conclude that the commonest cause of death, with or without vene- $\frac{0}{0}$ section, was liver failure. Only one patient in their $\underset{\mathbb{D}}{\stackrel{O}{0}}$ treated group of forty patients presented with $a \frac{\Omega}{\circ}$ dysrhythmia and heart failure. 
Repeated venesection removes excess iron and has been shown to reduce reactive fibrosis in the liver (Williams et al., 1969). However, only one of our patients (M.T.) showed improvement in the electrocardiograph after venesection. In two patients symptoms of angina became increasingly severe and the electrocardiographs suggested progressive myocardial damage, although in each case excess iron appeared to have been removed successfully. These changes may have been due to coronary artery disease as this is quite common in patients with haemochromatosis (Stocks and Powell, 1973), however, Swan and Dewar's description of one patient and their review of literature suggested that changes in the coronary arteries were not sufficient to account for the atrophy of muscles and fibrosis of the myocardium.

Cardiac failure does not appear to be a major presenting feature nor the commonest cause of death in this condition. Death is most commonly the result of hepatic failure. Liver cell carcinoma is well recognized as a cause of death in haemochromatosis, but secondary spread to involve the myocardium would appear to be very unusual and almost certainly unrelated to the electrocardiographic changes observed.

The discovery of an abnormal electrocardiogram may be the earliest manifestation of myocardial involvement in haemochromatosis and since there is evidence that with active treatment the changes may be reversible (McAllen, Coghill and Lubran, 1957), every patient with this condition should be followed with electrocardiographic recordings at regular intervals.

\section{Acknowledgments}

We are greatly indebted to Dr G. O. Richardson, Consultant Physician, and Dr B. Smith, Consultant Pathologist, for their contributions; Dr F. Clark, Consultant Physician and Dr E. H. Jarvis, Consultant Geriatric Physician for their kind permission to publish the cases, and Mrs O. Johnson for her assistance.

We would also like to thank the Photographic Department of the University for their co-operation.

\section{References}

British Medical Journal (1973) Leading Article. British Medical Journal, 2, 191.

FriedBerG, C.K. (1966) Diseases of the Heart, 3rd Edition. W. B. Saunders Company.

JAMES, T.N. (1964) Pathology of the cardiac conduction system in haemochromatosis. New England Journal Medicine, 271, 92.

McAllen, P.M., Coghill, N.F. \& Lubran, M. (1957) The treatment of haemochromatosis with particular reference to the removal of iron from the body by repeated venesection. Quarterly Journal of Medicine, 26, 251.

Schellhammer, P.F., Engle, M.A. \& Hangstorm, J.W.C. (1966) Arrhythmias, conduction defects and cardiac lesions in acquired iron storage disease. Circulation, Suppl. III to $33,34,208$.

Scheuer, P.J., Williams, R. \& Muir, A.R. (1962) Hepatic pathology in relatives of patients with haemochromatosis. Journal of Pathology and Bacteriology, 84, 1, 53.

SkinNer, C. \& Kenmure, A.C.F. (1973) Haemochromatosis presenting as congestive cardiomyopathy and responding to venesection. British Heart Journal, 35, 446.

Stocks, A.E. \& Powell, L.W. (1973) Carbohydrate intolerance in idiopathic haemochromatosis and cirrhosis of the liver. Quarterly Journal of Medicine, 168, 733.

SwaN, W.G.S. \& Dewar, H.A. (1952) The heart in haemochromatosis. British Heart Journal, 14, 117.

Thompson, R.B. (1969) Short Textbook of Haematology, 3rd Edition. Pitman Medical and Scientific Publications.

Truelove, S.C. \& Reynell, P.C. (1972) Diseases of the Digestive System, 2nd Edition. Blackwell Scientific Publications: Oxford.

Williams, R., Smith, P.M., Spicer, E.J.F., Barry, B. \& SHERLOCK, S. (1969) Venesection therapy in idiopathic haemochromatosis. Quarterly Journal of Medicine, 38, 1. 